International Journal of Pure and Applied Mathematics

Volume 86 No. 2 2013, 257-282

ISSN: 1311-8080 (printed version); ISSN: 1314-3395 (on-line version)

url: http://www.ijpam.eu

doi: http://dx.doi.org/10.12732/ijpam.v86i2.3

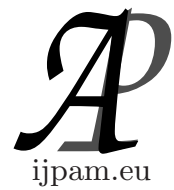

\title{
A STRENGTHENED FORM OF A GENERAL HARDY-TYPE INEQUALITY OBTAINED VIA SUPERQUADRATICITY AND ITS APPLICATIONS
}

\author{
Aleksandra Čižmešija ${ }^{1}$, Mario Krnić ${ }^{\S}$ \\ Department of Mathematics \\ University of Zagreb \\ Bijenička cesta 30, 10000, Zagreb, CROATIA \\ Faculty of Electrical Engineering and Computing \\ University of Zagreb \\ Unska 3, 10000, Zagreb, CROATIA
}

\begin{abstract}
In this paper, we establish a new refined version of the general Hardy-type inequality utilizing the notion of superquadratic functions. The general result obtained is then applied to some particular one-dimensional and multidimensional settings providing generalizations and refinements of some well-known Hardy-type inequalities. Finally, as another consequence of the main result, we also state and prove a class of improved versions of some HardyHilbert-type inequalities.
\end{abstract}

AMS Subject Classification: 26D10, 26D15

Key Words: Hardy inequality, Hardy-Hilbert inequality, Hardy-type inequalities, Hardy-Hilbert-type inequalities, non-conjugate exponents, superquadratic function, refinement, Riemann-Liouville operator, Weyl operator

\section{Introduction}

In 1925, Hardy stated and proved in [10] the integral inequality

Received: September 7, 2012

(c) 2013 Academic Publications, Ltd.

${ }^{\S}$ Correspondence author url: www.acadpubl.eu 


$$
\int_{0}^{\infty}\left(\frac{1}{x} \int_{0}^{x} f(y) d y\right)^{p} d x \leq\left(\frac{p}{p-1}\right)^{p} \int_{0}^{\infty} f^{p}(x) d x,
$$

where $p>1$ and $f$ is a non-negative function such that $\int_{0}^{\infty} f^{p}(x) d x<\infty$. This is the original form of the so-called Hardy integral inequality, which later on has been extensively studied and used as a model example for investigations of more general integral inequalities.

During subsequent decades, the Hardy inequality was generalized in many different ways. Roughly speaking, it was extended to what we call nowadays the Hardy-type inequality,

$$
\left[\int_{a}^{b} u(x)\left(\int_{a}^{x} f(y) d y\right)^{q} d x\right]^{\frac{1}{q}} \leq C_{p, q}\left(\int_{a}^{b} v(x) f^{p}(x) d x\right)^{\frac{1}{p}}
$$

where $f$ is a non-negative function, the parameters $a, b, p$, and $q$ are such that $-\infty \leq a<b \leq \infty, 0<q \leq \infty$, and $1 \leq p \leq \infty$, and where $u$ and $v$ are given weight functions. The central problem in connection with the Hardy inequality is to determine conditions on the parameters $p$ and $q$ and on the weight functions $u$ and $v$ under which the inequality holds for a certain class of functions. For a comprehensive inspection on the Hardy inequality, including its history, proofs, generalizations and diverse applications, the reader is referred to monographs [17], [18], and [21] (see also [11]).

In this paper, we refer to a more general class of Hardy-type inequalities regarding convex functions. Let $0<p \leq q<\infty$ and let $\left(\Omega_{1}, \Sigma_{1}, \mu_{1}\right)$ and $\left(\Omega_{2}, \Sigma_{2}, \mu_{2}\right)$ be measure spaces with positive $\sigma$-finite measures. Let $u: \Omega_{1} \rightarrow \mathbb{R}$ and $k: \Omega_{1} \times \Omega_{2} \rightarrow \mathbb{R}$ be non-negative measurable functions such that

$$
K(x)=\int_{\Omega_{2}} k(x, y) d \mu_{2}(y)>0, x \in \Omega_{1},
$$

and

$$
v(y)=\left[\int_{\Omega_{1}} u(x)\left(\frac{k(x, y)}{K(x)}\right)^{\frac{q}{p}} d \mu_{1}(x)\right]^{\frac{p}{q}}<\infty, y \in \Omega_{2} .
$$

In the above setting, Krulic et al. [16] proved that the general Hardy-type inequality

$$
\left(\int_{\Omega_{1}} u(x) \Psi^{\frac{q}{p}}\left(\mathcal{A}_{k} f(x)\right) d \mu_{1}(x)\right)^{\frac{1}{q}} \leq\left(\int_{\Omega_{2}} v(y) \Psi(f(y)) d \mu_{2}(y)\right)^{\frac{1}{p}}
$$

holds for all non-negative convex functions $\Psi$ defined on a convex set $I \subseteq \mathbb{R}$, all measurable functions $f: \Omega_{2} \rightarrow \mathbb{R}$ such that $f\left(\Omega_{2}\right) \subseteq I$, and the general integral 
operator $\mathcal{A}_{k}$ defined by

$$
\mathcal{A}_{k} f(x)=\frac{1}{K(x)} \int_{\Omega_{2}} k(x, y) f(y) d \mu_{2}(y), x \in \Omega_{1} .
$$

Observe that inequality (4) generalizes the classical Hardy inequality (1). Namely, let $\Omega_{1}=\Omega_{2}=\mathbb{R}_{+}=(0, \infty), d \mu_{1}(x)=d x, d \mu_{2}(y)=d y$, and $u(x)=\frac{1}{x}$. If $1<p=q<\infty$ and $\Psi:[0, \infty) \rightarrow \mathbb{R}$ is defined by $\Psi(x)=x^{p}$, then (1) follows directly from (4), rewritten with the kernel $k(x, y)=\frac{1}{x} \chi_{0<y \leq x<\infty}(x, y)$ and with $f\left(y^{\frac{p}{p-1}}\right) y^{\frac{1}{p-1}}$ instead of $f(y)$. In the same setting, except with $f(y) y^{\frac{1}{p}}$ instead of $f(y)$, and with $k(x, y)=\left(\frac{x}{y}\right)^{\frac{1}{p}}(x+y)^{-1}$, relation (4) becomes the well-known Hardy-Hilbert integral inequality

$$
\int_{0}^{\infty}\left(\int_{0}^{\infty} \frac{f(y)}{x+y} d y\right)^{p} d x \leq\left(\frac{\pi}{\sin \frac{\pi}{p}}\right)^{p} \int_{0}^{\infty} f^{p}(x) d x
$$

for a non-negative function $f \in L^{p}\left(\mathbb{R}_{+}\right)$.

Nowadays, a considerable attention has been given to developing methods for improving the above mentioned Hardy-type inequalities. One such approach involves superquadratic functions, a class of functions closely connected to convex functions (see the next section). Considering a superquadratic function instead of a convex function $\Psi$, Abramovich et al. [4] obtained the following refinement of inequality (4) in the particular case when $p=q$ :

$$
\begin{aligned}
& \int_{\Omega_{1}} u(x) \Psi\left(\mathcal{A}_{k} f(x)\right) d \mu_{1}(x) \\
& \quad+\int_{\Omega_{1}} \int_{\Omega_{2}} u(x) \frac{k(x, y)}{K(x)} \Psi\left(\left|f(y)-\mathcal{A}_{k} f(x)\right|\right) d \mu_{1}(x) d \mu_{2}(y) \\
& \leq \int_{\Omega_{2}} v(y) \Psi(f(y)) d \mu_{2}(y),
\end{aligned}
$$

where

$$
v(y)=\int_{\Omega_{1}} u(x) \frac{k(x, y)}{K(x)} d \mu_{1}(x)<\infty, \quad y \in \Omega_{2} .
$$

Our main intention in this paper is to derive a refinement of the general Hardy-type inequality (4) for all admissible parameters $p$ and $q$, covering the particular case (7), what can be done by virtue of superquadraticity. Moreover, applying the general result obtained to some particular classical and new onedimensional and multidimensional settings, we derive several classes of new refined inequalities of the Hardy and the Hardy-Hilbert type. 
The paper is organized in the following way: after this Introduction, in Section 2, we recall the notion and some basic properties of superquadratic functions. Further, in Section 3, we state and prove our basic result, that is, a refinement of inequality (4) by exploiting superquadraticity. Sections 4 and 5 are dedicated to some particular one-dimensional and multidimensional examples arising from the general result and yielding improved versions of numerous results known from the literature. Finally, in Section 6, we also obtain a strengthened version of a class of the Hardy-Hilbert-type inequalities involving a homogeneous kernel of a negative real degree.

\section{Preliminaries}

First, let us introduce some conventions used throughout this paper (if nothing else is explicitly stated). Namely, all functions are assumed to be measurable and expressions of the form $0 \cdot \infty, \frac{0}{0}, \frac{\infty}{\infty}$, and $\frac{a}{\infty}$, where $a \in \mathbb{R}$, are taken to be equal to zero. Moreover, all integrals are assumed to converge. For a real parameter $0 \neq p \neq 1$, by $p^{\prime}$ we denote its conjugate exponent $p^{\prime}=\frac{p}{p-1}$, that is, $\frac{1}{p}+\frac{1}{p^{\prime}}=1$. For $p>0$, by $L^{p}\left(\mathbb{R}_{+}\right)$we denote the usual Lebesgue space. In addition, by a weight function (shortly: a weight) we mean a non-negative measurable function on the actual set. Further, $\log x$ is the natural logarithm of $x \in \mathbb{R}_{+}$, while $B(\cdot ; \cdot, \cdot)$ denotes the incomplete Beta function, defined by

$$
B(x ; a, b)=\int_{0}^{x} t^{a-1}(1-t)^{b-1} d t, \quad x \in[0,1], a, b>0 .
$$

As usual, $B(a, b)=B(1 ; a, b)$ stands for the standard Beta function. Finally, $\Gamma(\cdot)$ denotes the usual Gamma function defined by

$$
\Gamma(a)=\int_{0}^{\infty} t^{a-1} e^{-t} d t, \quad a>0 .
$$

For reader convenience, we also recall the definition and some basic properties of superquadratic functions, introduced by Abramovich et al. [2] (for more information see also $[1,3])$. A function $\Psi:[0, \infty) \rightarrow \mathbb{R}$ is called superquadratic provided that for each $x \geq 0$ there exists a constant $C_{x} \in \mathbb{R}$ such that

$$
\Psi(y)-\Psi(x)-\Psi(|y-x|) \geq C_{x}(y-x)
$$

holds for all $y \geq 0$.

Superquadratic functions are closely related to convex functions. In particular, at the first sight, condition (8) appears to be stronger than convexity. 
However, if $\Psi$ takes negative values, it may be considerably weaker. Just to see how poorly behaved superquadratic functions can be, we remark that any function $\Psi$ with values in the closed interval $[-2,-1]$ is superquadratic. This conclusion simply follows by observing that the left-hand side of (8) takes values in $[0,3]$ in that case and then by putting $C_{x}=0$. On the other hand, non-negative superquadratic functions are much better behaved, namely, they are convex.

It is also well-known that a continuously differentiable function $\Psi:[0, \infty) \rightarrow$ $\mathbb{R}$, such that $\Psi(0) \leq 0$, is superquadratic if the function $x \mapsto \frac{\Psi^{\prime}(x)}{x}$ is nondecreasing on $\mathbb{R}_{+}$or the function $\Psi^{\prime}$ is superadditive, that is, $\Psi^{\prime}(x+y) \geq$ $\Psi^{\prime}(x)+\Psi^{\prime}(y), x, y \geq 0$ (see [2, Lemma 3.1]). As a consequence, the power function $\Psi:[0, \infty) \rightarrow \mathbb{R}, \Psi(x)=x^{p}$, is superquadratic for all $p \in \mathbb{R}_{+}, p \geq 2$.

In addition, another important characterization of a superquadratic function is the following version of the refined Jensen inequality:

$$
\begin{gathered}
\Psi\left(\int_{\Omega} f(x) d \mu(x)\right)+\int_{\Omega} \Psi\left(\left|f(x)-\int_{\Omega} f(x) d \mu(x)\right|\right) d \mu(x) \\
\leq \int_{\Omega} \Psi(f(x)) d \mu(x) .
\end{gathered}
$$

It holds for all probability measure spaces $(\Omega, \Sigma, \mu)$ and all non-negative $\mu$ integrable functions $f$ on $\Omega$ if and only if $\Psi:[0, \infty) \rightarrow \mathbb{R}$ is a superquadratic function (see [2, Theorem 2.3]). The above inequality (9) will be crucial in establishing our new refinement of the general Hardy-type inequality (4).

\section{The Main Result}

Handling with superquadratic functions appears to be quite fruitful in improving Hardy-type inequalities. The first particular results in this direction are due to Oguntuase, Persson, Essel and Popoola (see papers [19, 20, 22]) and the crucial step in their obtaining was in applying the refined Jensen inequality (9). Moreover, as already mentioned, Abramovich et al. [4], derived a refinement (7) of inequality (4) in the case when $\Psi$ is a superquadratic function and $p=q$. This result will be obtained as a simple consequence of our main result below.

Motivated by the above mentioned results, our main intention is to establish a refinement of the general Hardy-type inequality (4), covering all admissible parameters $p$ and $q$ and including (7). It will also be derived by exploiting the refined Jensen inequality (9). In the sequel, we deal with non-negative 
superquadratic functions. Since such functions are convex, the following result holds for this particular subclass of convex functions.

Theorem 1. Let $\left(\Omega_{1}, \Sigma_{1}, \mu_{1}\right),\left(\Omega_{2}, \Sigma_{2}, \mu_{2}\right)$ be measure spaces with positive $\sigma$-finite measures and let $u: \Omega_{1} \rightarrow \mathbb{R}$ and $k: \Omega_{1} \times \Omega_{2} \rightarrow \mathbb{R}$ be non-negative measurable functions. Further, suppose that $K: \Omega_{1} \rightarrow \mathbb{R}$ is defined as in (3) and

$$
v(y)=\left[\int_{\Omega_{1}} u(x)\left(\frac{k(x, y)}{K(x)}\right)^{r} d \mu_{1}(x)\right]^{\frac{1}{r}}<\infty, \quad y \in \Omega_{2},
$$

where $r \geq 1$. If $\Psi:[0, \infty) \rightarrow \mathbb{R}$ is a non-negative superquadratic function, then the inequality

$$
\begin{aligned}
\int_{\Omega_{1}} u(x) \Psi^{r}\left(\mathcal{A}_{k} f(x)\right) d \mu_{1}(x) \\
\quad+r \int_{\Omega_{1}} \int_{\Omega_{2}} u(x) \frac{k(x, y)}{K(x)} \Psi^{r-1}\left(\mathcal{A}_{k} f(x)\right) \Psi\left(\left|f(y)-\mathcal{A}_{k} f(x)\right|\right) d \mu_{1}(x) d \mu_{2}(y) \\
\leq\left(\int_{\Omega_{2}} v(y) \Psi(f(y)) d \mu_{2}(y)\right)^{r}
\end{aligned}
$$

holds for all non-negative measurable functions $f: \Omega_{2} \rightarrow \mathbb{R}$ and for $\mathcal{A}_{k} f: \Omega_{1} \rightarrow$ $\mathbb{R}$ defined by (5).

Proof. Applying inequality (9) to the superquadratic function $\Psi$, for each fixed $x \in \Omega_{1}$ we get

$$
\begin{gathered}
\Psi\left(\mathcal{A}_{k} f(x)\right)+\frac{1}{K(x)} \int_{\Omega_{2}} k(x, y) \Psi\left(\left|f(y)-\mathcal{A}_{k} f(x)\right|\right) d \mu_{2}(y) \\
\leq \frac{1}{K(x)} \int_{\Omega_{2}} k(x, y) \Psi(f(y)) d \mu_{2}(y)
\end{gathered}
$$

wherefrom

$$
\begin{gathered}
\left(\Psi\left(\mathcal{A}_{k} f(x)\right)+\frac{1}{K(x)} \int_{\Omega_{2}} k(x, y) \Psi\left(\left|f(y)-\mathcal{A}_{k} f(x)\right|\right) d \mu_{2}(y)\right)^{r} \\
\leq\left(\frac{1}{K(x)} \int_{\Omega_{2}} k(x, y) \Psi(f(y)) d \mu_{2}(y)\right)^{r}
\end{gathered}
$$

since $r \geq 1$. Further, by virtue of the well-known Bernoulli inequality, it follows that the left-hand side of inequality (12) is not less than

$$
\Psi^{r}\left(\mathcal{A}_{k} f(x)\right)+r \frac{\Psi^{r-1}\left(\mathcal{A}_{k} f(x)\right)}{K(x)} \int_{\Omega_{2}} k(x, y) \Psi\left(\left|f(y)-\mathcal{A}_{k} f(x)\right|\right) d \mu_{2}(y),
$$


that is, we obtain the inequality

$$
\begin{aligned}
\Psi^{r}\left(\mathcal{A}_{k} f(x)\right)+ & r \frac{\Psi^{r-1}\left(\mathcal{A}_{k} f(x)\right)}{K(x)} \int_{\Omega_{2}} k(x, y) \Psi\left(\left|f(y)-\mathcal{A}_{k} f(x)\right|\right) d \mu_{2}(y) \\
& \leq\left(\frac{1}{K(x)} \int_{\Omega_{2}} k(x, y) \Psi(f(y)) d \mu_{2}(y)\right)^{r} .
\end{aligned}
$$

Now, multiplying the above inequality $(13)$ by $u(x)$ and integrating over $\Omega_{1}$, we have

$$
\begin{aligned}
& \int_{\Omega_{1}} u(x) \Psi^{r}\left(\mathcal{A}_{k} f(x)\right) d \mu_{1}(x) \\
& +r \int_{\Omega_{1}} \frac{u(x) \Psi^{r-1}\left(\mathcal{A}_{k} f(x)\right)}{K(x)}\left(\int_{\Omega_{2}} k(x, y) \Psi\left(\left|f(y)-\mathcal{A}_{k} f(x)\right|\right) d \mu_{2}(y)\right) d \mu_{1}(x) \\
& \leq \int_{\Omega_{1}} u(x)\left(\frac{1}{K(x)} \int_{\Omega_{2}} k(x, y) \Psi(f(y)) d \mu_{2}(y)\right)^{r} d \mu_{1}(x)
\end{aligned}
$$

Finally, utilizing the Minkowski inequality and taking into account definition (10) of the weight function $v$, we obtain that the right-hand side of (14) is not greater than

$$
\begin{gathered}
{\left[\int_{\Omega_{2}} \Psi(f(y))\left(\int_{\Omega_{1}} u(x)\left(\frac{k(x, y)}{K(x)}\right)^{r} d \mu_{1}(x)\right)^{\frac{1}{r}} d \mu_{2}(y)\right]^{r}} \\
=\left(\int_{\Omega_{2}} v(y) \Psi(f(y)) d \mu_{2}(y)\right)^{r}
\end{gathered}
$$

The proof is now completed.

Remark 1. Since $\Psi$ is a non-negative superquadratic function, the second term on the left-hand side of inequality (11) is non-negative. Hence, relation (11) with $r=\frac{q}{p}$ provides a refinement of the general Hardy-type inequality (4).

Remark 2. Observe that Theorem 1 holds also for a non-negative superquadratic function $\Psi$ on an interval (that is, a convex set) $I \subseteq \mathbb{R}_{+}$, provided that $f: \Omega_{2} \rightarrow \mathbb{R}$ takes values in $I$. This conclusion is based on the fact that $\mathcal{A}_{k} f(x)$ is a generalized mean, so $f(x) \in I$ for all $x \in \Omega_{2}$ implies that $\mathcal{A}_{k} f(x)$ belongs to the interval $I$ as well. For a more formal proof of this fact, the reader is referred to [4].

Remark 3. Since for $r=1$ relation (11) reduces to (7), our Theorem 1 may be regarded as an extension of the corresponding result from [4]. 


\section{Refined One-Dimensional Hardy-Type Inequalities}

The main objective of this section is a study of one-dimensional consequences of Theorem 1, which represent further generalizations of the classical Hardy inequality (1), as well as of its dual form. More precisely, we consider here the usual one-dimensional settings with intervals in $\mathbb{R}$ and the Lebesgue measure.

First, suppose that $0<b \leq \infty$ and $S_{1}=\left\{(x, y) \in \mathbb{R}^{2}: 0<y \leq x<b\right\}$. Applying Theorem 1 to $\Omega_{1}=\Omega_{2}=(0, b), d \mu_{1}(x)=d x, d \mu_{2}(y)=d y$, and to $k \chi_{S_{1}}(x, y), \frac{u(x)}{x}$, and $\frac{w(y)}{y}$, respectively instead of $k(x, y), u(x)$, and $v(y)$, we immediately obtain its following important consequence.

Corollary 1. Let $0<b \leq \infty$ and $k:(0, b) \times(0, b) \rightarrow \mathbb{R}, u:(0, b) \rightarrow \mathbb{R}$ be non-negative measurable functions, such that

$$
K(x)=\int_{0}^{x} k(x, y) d y>0, \quad x \in(0, b),
$$

and let

$$
w(y)=y\left[\int_{y}^{b} u(x)\left(\frac{k(x, y)}{K(x)}\right)^{r} \frac{d x}{x}\right]^{\frac{1}{r}}<\infty, \quad y \in(0, b),
$$

where $r \geq 1$. If $\Psi:[0, \infty) \rightarrow \mathbb{R}$ is a non-negative superquadratic function, then the inequality

$$
\begin{aligned}
\int_{0}^{b} u(x) & \Psi^{r}\left(\mathcal{A}_{k} f(x)\right) \frac{d x}{x} \\
& +r \int_{0}^{b} \int_{0}^{x} u(x) \frac{k(x, y)}{K(x)} \Psi^{r-1}\left(\mathcal{A}_{k} f(x)\right) \Psi\left(\left|f(y)-\mathcal{A}_{k} f(x)\right|\right) \frac{d x}{x} d y \\
\leq & \left(\int_{0}^{b} w(y) \Psi(f(y)) \frac{d y}{y}\right)^{r}
\end{aligned}
$$

holds for all non-negative measurable functions $f:(0, b) \rightarrow \mathbb{R}$ and for the operator

$$
\mathcal{A}_{k} f(x)=\frac{1}{K(x)} \int_{0}^{x} k(x, y) f(y) d y, \quad x \in(0, b) .
$$

On the other hand, consider the set $S_{2}=\left\{(x, y) \in \mathbb{R}^{2}: b<x \leq y<\infty\right\}$, where $0 \leq b<\infty$. Then, setting $\Omega_{1}=\Omega_{2}=(b, \infty), d \mu_{1}(x)=d x, d \mu_{2}(y)=d y$, and replacing $k(x, y), u(x)$, and $v(y)$ respectively with $k \chi_{S_{2}}(x, y), \frac{u(x)}{x}$, and $\frac{w(y)}{y}$, Theorem 1 provides a dual form of Corollary 1. It is given in the following corollary. 
Corollary 2. Let $0 \leq b<\infty$ and $k:(b, \infty) \times(b, \infty) \rightarrow \mathbb{R}, u:(b, \infty) \rightarrow \mathbb{R}$ be non-negative measurable functions such that

$$
\widetilde{K}(x)=\int_{x}^{\infty} k(x, y) d y>0, \quad x \in(b, \infty),
$$

and let

$$
\widetilde{w}(y)=y\left[\int_{b}^{y} u(x)\left(\frac{k(x, y)}{K(x)}\right)^{r} \frac{d x}{x}\right]^{\frac{1}{r}}<\infty, \quad y \in(b, \infty),
$$

where $r \geq 1$. If $\Psi:[0, \infty) \rightarrow \mathbb{R}$ is a non-negative superquadratic function, then the inequality

$$
\begin{aligned}
\int_{b}^{\infty} & u(x) \Psi^{r}\left(\widetilde{\mathcal{A}}_{k} f(x)\right) \frac{d x}{x} \\
& +r \int_{b}^{\infty} \int_{x}^{\infty} u(x) \frac{k(x, y)}{\widetilde{K}(x)} \Psi^{r-1}\left(\widetilde{\mathcal{A}}_{k} f(x)\right) \Psi\left(\left|f(y)-\widetilde{\mathcal{A}}_{k} f(x)\right|\right) \frac{d x}{x} d y \\
\leq & \left(\int_{b}^{\infty} \widetilde{w}(y) \Psi(f(y)) \frac{d y}{y}\right)^{r}
\end{aligned}
$$

holds for all non-negative measurable functions $f:(b, \infty) \rightarrow \mathbb{R}$ and for the operator

$$
\widetilde{\mathcal{A}}_{k} f(x)=\frac{1}{\widetilde{K}(x)} \int_{x}^{\infty} k(x, y) f(y) d y, \quad x \in(b, \infty) .
$$

Corollaries 1 and 2 cover and refine numerous Hardy-type inequalities known from the literature. To corroborate our assertion, we consider several examples with particular kernels and weight functions. One of the most interesting consequences of Corollary 1 is related to the so-called Riemann-Liouville operator

$$
\mathcal{R}_{\gamma} f(x)=\frac{\gamma}{x^{\gamma}} \int_{0}^{x}(x-y)^{\gamma-1} f(y) d y, \quad x \in(0, b),
$$

considered in the following example.

Example 1. Considering Corollary 1 with the functions $k:(0, b) \times(0, b) \rightarrow$ $\mathbb{R}$ and $u:(0, b) \rightarrow \mathbb{R}$ respectively defined by $k(x, y)=\frac{\gamma}{x^{\gamma}}(x-y)^{\gamma-1} \chi_{S_{1}}(x, y)$ and $u(x) \equiv 1$, and providing that $\gamma>1-\frac{1}{r}$ and $r \geq 1$, it follows that $K(x) \equiv 1$ and the operator $\mathcal{A}_{k}$, defined by (16), reduces to the above Riemann-Liouville operator $\mathcal{R}_{\gamma}$, given by (19). Moreover, in the described setting, inequality (15) reads

$$
\int_{0}^{b} \Psi^{r}\left(\mathcal{R}_{\gamma} f(x)\right) \frac{d x}{x}
$$




$$
\begin{aligned}
& +r \gamma \int_{0}^{b} \int_{0}^{x}(x-y)^{\gamma-1} \Psi^{r-1}\left(\mathcal{R}_{\gamma} f(x)\right) \Psi\left(\left|f(y)-\mathcal{R}_{\gamma} f(x)\right|\right) \frac{d x}{x^{\gamma+1}} d y \\
\leq & \left(\int_{0}^{b} w_{\gamma}(y) \Psi(f(y)) \frac{d y}{y}\right)^{r},
\end{aligned}
$$

where the weight function $w_{\gamma}$ is expressed in terms of the incomplete Beta function, that is,

$$
w_{\gamma}(y)=\gamma\left(\int_{0}^{1-\frac{y}{b}} t^{(\gamma-1) r}(1-t)^{r-1} d t\right)^{\frac{1}{r}}=\gamma B^{\frac{1}{r}}\left(1-\frac{y}{b} ;(\gamma-1) r+1, r\right) .
$$

Now, in order to compare relation (20) with the classical Hardy inequality, we consider it with the superquadratic function $\Psi(x)=x^{p}$, the parameter $r=\frac{q}{p}$, where $q \geq p \geq 2$, and with $b$ and $f(y)$, respectively replaced with $b^{\frac{k-1}{p}}$ and $f\left(y^{\frac{p}{k-1}}\right) y^{\frac{p}{k-1}-1}, k>1$. After a sequence of a suitable variable changes, we obtain the following strengthened form of the Hardy-type inequality:

$$
\begin{aligned}
& \int_{0}^{b} x^{\frac{q}{p}(1-k)-1}(\mathcal{R} f)^{q}(x) d x+\frac{\gamma q(k-1)}{p^{2}} \int_{0}^{b} \int_{y}^{b}\left[1-\left(\frac{y}{x}\right)^{\frac{k-1}{p}}\right]^{\gamma-1}(\mathcal{R} f)^{q-p}(x) \\
& \quad \times\left|\frac{p}{\gamma(k-1)} f(y)\left(\frac{y}{x}\right)^{1-\frac{k-1}{p}}-\frac{1}{x} \mathcal{R} f(x)\right|^{p} x^{\frac{k-1}{p}(p-q-1)+p-k} y^{\frac{k-1}{p}-1} d x d y \\
& \leq \gamma\left(\frac{p}{\gamma(k-1)}\right)^{q+1-\frac{q}{p}}\left(\int_{0}^{b} w_{\gamma, k}(y) f^{p}(y) d y\right)^{\frac{q}{p}}
\end{aligned}
$$

with the operator $\mathcal{R}$ and the weight function $w_{\gamma, k}$ defined by

$$
\mathcal{R} f(x)=\int_{0}^{x}\left[1-\left(\frac{y}{x}\right)^{\frac{k-1}{p}}\right]^{\gamma-1} f(y) d y, \quad x \in(0, b),
$$

and

$$
w_{\gamma, k}(y)=B^{\frac{p}{q}}\left(1-\left(\frac{y}{b}\right)^{\frac{k-1}{p}} ;(\gamma-1) \frac{q}{p}+1, \frac{q}{p}\right) y^{p-k}, \quad y \in(0, b) .
$$

Remark 4. Observe that for $k=q=p \geq 2$ and $\gamma=1$, relation (21) reads

$$
\begin{aligned}
\int_{0}^{b}(\mathcal{H} f)^{p}(x) d x & +\frac{p-1}{p} \int_{0}^{b} \int_{y}^{b}\left|\frac{p}{p-1} f(y)\left(\frac{y}{x}\right)^{\frac{1}{p}}-\mathcal{H} f(x)\right|^{p} x^{\frac{1}{p}-1} y^{-\frac{1}{p}} d x d y \\
& \leq\left(\frac{p}{p-1}\right)^{p} \int_{0}^{b}\left[1-\left(\frac{y}{b}\right)^{\frac{p-1}{p}}\right] f^{p}(y) d y
\end{aligned}
$$


where $\mathcal{H}$ is the classical Hardy (or Cesaro) operator

$$
\mathcal{H} f(x)=\frac{1}{x} \int_{0}^{x} f(y) d y .
$$

It should be noticed that for $b=\infty$ the above relation (22) represents a refinement of the classical Hardy inequality (1).

Remark 5. Another interesting feature in connection with Example 1 appears when considering (20) with $r=\gamma=1$ and $b=\infty$. In this setting, inequality (20) becomes

$$
\int_{0}^{\infty} \Psi(\mathcal{H} f(x)) \frac{d x}{x}+\int_{0}^{\infty} \int_{0}^{x} \Psi(|f(y)-\mathcal{H} f(x)|) \frac{d x}{x^{2}} d y \leq \int_{0}^{\infty} \Psi(f(y)) \frac{d y}{y},
$$

providing a refinement of the inequality

$$
\int_{0}^{\infty} \Psi(\mathcal{H} f(x)) \frac{d x}{x} \leq \int_{0}^{\infty} \Psi(f(y)) \frac{d y}{y},
$$

when $\Psi$ is a non-negative superquadratic function. Inequality (24) was extensively studied in paper [12], but was known even before (see e.g. Godunova $[7])$.

The following consequence of Corollary 2, related to the so-called Weyl operator

$$
\mathcal{W}_{\gamma} f(x)=\gamma x \int_{x}^{\infty}(y-x)^{\gamma-1} f(y) \frac{d y}{y^{\gamma+1}}, \quad x \in(b, \infty),
$$

provides a refined dual form of the Hardy inequality.

Example 2. Similarly to Example 1, we consider now inequality (17) with the kernel $k:(b, \infty) \times(b, \infty) \rightarrow \mathbb{R}, k(x, y)=\gamma \frac{x}{y^{\gamma+1}}(y-x)^{\gamma-1} \chi_{S_{2}}(x, y)$, and the weight function $u:(b, \infty) \rightarrow \mathbb{R}, u(x) \equiv 1$, provided that $\gamma>1-\frac{1}{r}$ and $r \geq 1$. In this setting, we have $\widetilde{K}(x) \equiv 1$ and the operator $\widetilde{\mathcal{A}}_{k}$, defined by (18), reduces to the Weyl operator $\mathcal{W}_{\gamma}$ given by (25). Therefore, inequality (17) can be rewritten in the form

$$
\begin{aligned}
\int_{b}^{\infty} & \Psi^{r}\left(\mathcal{W}_{\gamma} f(x)\right) \frac{d x}{x} \\
& +r \gamma \int_{b}^{\infty} \int_{x}^{\infty}(y-x)^{\gamma-1} \Psi^{r-1}\left(\mathcal{W}_{\gamma} f(x)\right) \Psi\left(\left|f(y)-\mathcal{W}_{\gamma} f(x)\right|\right) d x \frac{d y}{y^{\gamma+1}} \\
\leq & \left(\int_{b}^{\infty} \widetilde{w}_{\gamma}(y) \Psi(f(y)) \frac{d y}{y}\right)^{r}
\end{aligned}
$$


where $\widetilde{w}_{\gamma}$ is expressed in terms of the incomplete Beta function,

$$
\widetilde{w}_{\gamma}(y)=\gamma\left(\int_{0}^{1-\frac{b}{y}} t^{(\gamma-1) r}(1-t)^{r-1} d t\right)^{\frac{1}{r}}=\gamma B^{\frac{1}{r}}\left(1-\frac{b}{y} ;(\gamma-1) r+1, r\right) .
$$

In addition, the classical form of the dual Hardy inequality arises when relation (26) is considered with the superquadratic function $\Psi(x)=x^{p}$, the parameter $r=\frac{q}{p}$, where $q \geq p \geq 2$, and with $b$ and $f(y)$ respectively substituted with $b^{\frac{1-k}{p}}$ and $f\left(y^{\frac{p}{1-k}}\right) y^{\frac{p}{1-k}+1}, k<1$. More precisely, by exploiting several suitable variable changes, we obtain a refinement of the dual Hardy-type inequality,

$$
\begin{aligned}
& \int_{b}^{\infty} x^{\frac{q}{p}(1-k)-1}(\mathcal{W} f)^{q}(x) d x \\
& \quad+\frac{\gamma q(1-k)}{p^{2}} \int_{b}^{\infty} \int_{b}^{y}\left[1-\left(\frac{x}{y}\right)^{\frac{1-k}{p}}\right]^{\gamma-1}(\mathcal{W} f)^{q-p}(x) \\
& \quad \times\left|\frac{p}{\gamma(1-k)} f(y)\left(\frac{y}{x}\right)^{1+\frac{1-k}{p}}-\frac{1}{x} \mathcal{W} f(x)\right|^{p} x^{\frac{1-k}{p}(q-p+1)+p-k} y^{-\frac{1-k}{p}-1} d x d y \\
& \leq \gamma\left(\frac{p}{\gamma(1-k)}\right)^{q+1-\frac{q}{p}}\left(\int_{b}^{\infty} \widetilde{w}_{\gamma, k}(y) f^{p}(y) d y\right)^{\frac{q}{p}}
\end{aligned}
$$

where the operator $\mathcal{W}$ and the weight function $\widetilde{w}_{\gamma, k}$ are respectively defined by

$$
\mathcal{W} f(x)=\int_{x}^{\infty}\left[1-\left(\frac{x}{y}\right)^{\frac{1-k}{p}}\right]^{\gamma-1} f(y) d y, \quad x \in(b, \infty),
$$

and

$$
\widetilde{w}_{\gamma, k}(y)=B^{\frac{p}{q}}\left(1-\left(\frac{b}{y}\right)^{\frac{1-k}{p}} ;(\gamma-1) \frac{q}{p}+1, \frac{q}{p}\right) y^{p-k}, \quad y \in(b, \infty) .
$$

Remark 6. If $p=q$ and $\gamma=1$, relations (21) and (27) reduce to the corresponding inequalities obtained in paper [19].

\section{Refined Multidimensional Hardy-Type Inequalities}

The method of refining the Hardy-type inequalities via superquadraticity, developed in this paper, can also be applied to some multidimensional settings. 
In fact, one-dimensional examples derived in the previous section can naturally be extended to a multidimensional level.

Before stating the corresponding results, we introduce some notation. First, we set $\mathbf{0}=(0,0, \ldots, 0), \mathbf{1}=(1,1, \ldots, 1) \in \mathbb{R}^{n}$, and for $\mathbf{x}=\left(x_{1}, x_{2}, \ldots, x_{n}\right) \in$ $\mathbb{R}_{+}^{n}$ and $\mathbf{y}=\left(y_{1}, y_{2}, \ldots, y_{n}\right) \in \mathbb{R}^{n}$ denote

$$
\frac{\mathbf{y}}{\mathbf{x}}=\left(\frac{y_{1}}{x_{1}}, \frac{y_{2}}{x_{2}}, \ldots, \frac{y_{n}}{x_{n}}\right) \quad \text { and } \quad \mathbf{x}^{\mathbf{y}}=x_{1}^{y_{1}} x_{2}^{y_{2}} \cdot \ldots \cdot x_{n}^{y_{n}} .
$$

In particular, $\mathbf{x}^{\mathbf{1}}=\prod_{i=1}^{n} x_{i}$. Further, for $\mathbf{x}, \mathbf{y} \in \mathbb{R}^{n}$ we write $\mathbf{x}<\mathbf{y}$ if componentwise $x_{i}<y_{i}, i=1, \ldots, n$, and relations $\leq,>$, and $\geq$ are defined analogously. For $\mathbf{a}, \mathbf{b} \in \mathbb{R}^{n}, \mathbf{a}<\mathbf{b}$, we define $(\mathbf{a}, \mathbf{b})=\left\{\mathbf{x} \in \mathbb{R}^{n}: \mathbf{a}<\mathbf{x}<\mathbf{b}\right\}$. Moreover,

$$
(\mathbf{a}, \infty)=\left\{\mathbf{x} \in \mathbb{R}^{n}: \mathbf{a}<\mathbf{x}<\infty\right\}
$$

and the $n$-cells $[\mathbf{a}, \mathbf{b}),(\mathbf{a}, \mathbf{b}]$ and $[\mathbf{a}, \mathbf{b}]$ are defined similarly. Finally, the integral $\int_{(\mathbf{a}, \mathbf{b})} f(\mathbf{x}) d \mathbf{x}$ is interpreted as $\int_{a_{1}}^{b_{1}} \int_{a_{2}}^{b_{2}} \ldots \int_{a_{n}}^{b_{n}} f\left(x_{1}, x_{2}, \ldots, x_{n}\right) d x_{1} \ldots d x_{n}$.

Now, suppose that $\Omega_{1}=\Omega_{2}=\mathbb{R}_{+}^{n}$ with the corresponding Lebesgue measures $d \mu_{1}(\mathbf{x})=d \mathbf{x}$ and $d \mu_{2}(\mathbf{y})=d \mathbf{y}$. As distinguished from the previous section, we deal here with a slightly altered form of the kernel $k$. More precisely, the kernel $k: \mathbb{R}_{+}^{n} \times \mathbb{R}_{+}^{n} \rightarrow \mathbb{R}$ is assumed to be of the form $k(\mathbf{x}, \mathbf{y})=l\left(\frac{\mathbf{y}}{\mathbf{x}}\right)$, where $l: \mathbb{R}_{+}^{n} \rightarrow \mathbb{R}$ is a non-negative measurable function. The first Hardy-type inequality in the above setting was established by Godunova. In fact, it was a multidimensional version of inequality (24) (for more details see [8]).

Our main intention here is to derive a relation that generalizes and improves the above mentioned Godunova's result. However, this can be done by virtue of our general result. More precisely, applying Theorem 1 to the multidimensional setting described and to $u(\mathbf{x})$ and $v(\mathbf{y})$ respectively replaced with $\frac{u(\mathbf{x})}{\mathbf{x}^{1}}$ and $\frac{w(\mathbf{y})}{\mathbf{y}^{1}}$, we obtain the following Hardy-type inequality.

Theorem 2. Let $u: \mathbb{R}_{+}^{n} \rightarrow \mathbb{R}$ and $l: \mathbb{R}_{+}^{n} \rightarrow \mathbb{R}$ be non-negative measurable functions such that $L(\mathbf{x})=\mathbf{x}^{1} \int_{\mathbb{R}_{+}^{n}} l(\mathbf{y}) d \mathbf{y}>0$ for all $\mathbf{x} \in \mathbb{R}_{+}^{n}$. Further, let the function $w: \mathbb{R}_{+}^{n} \rightarrow \mathbb{R}$ be defined as

$$
w(\mathbf{y})=\mathbf{y}^{\mathbf{1}}\left[\int_{\mathbb{R}_{+}^{n}} u(\mathbf{x})\left(\frac{l\left(\frac{\mathbf{y}}{\mathbf{x}}\right)}{L(\mathbf{x})}\right)^{r} \frac{d \mathbf{x}}{\mathbf{x}^{\mathbf{1}}}\right]^{\frac{1}{r}},
$$

where $r \geq 1$. If $\Psi:[0, \infty) \rightarrow \mathbb{R}$ is a non-negative superquadratic function, then the inequality

$$
\int_{\mathbb{R}_{+}^{n}} u(\mathbf{x}) \Psi^{r}\left(\mathcal{A}_{l} f(\mathbf{x})\right) \frac{d \mathbf{x}}{\mathbf{x}^{1}}
$$




$$
\begin{aligned}
& +r \int_{\mathbb{R}_{+}^{n}} \int_{\mathbb{R}_{+}^{n}} u(\mathbf{x}) \frac{l\left(\frac{\mathbf{y}}{\mathbf{x}}\right)}{L(\mathbf{x})} \Psi^{r-1}\left(\mathcal{A}_{l} f(\mathbf{x})\right) \Psi\left(\left|f(\mathbf{y})-\mathcal{A}_{l} f(\mathbf{x})\right|\right) \frac{d \mathbf{x}}{\mathbf{x}^{\mathbf{1}}} d \mathbf{y} \\
\leq & \left(\int_{\mathbb{R}_{+}^{n}} w(\mathbf{y}) \Psi(f(\mathbf{y})) \frac{d \mathbf{y}}{\mathbf{y}^{\mathbf{1}}}\right)^{r}
\end{aligned}
$$

holds for all non-negative measurable functions $f: \mathbb{R}_{+}^{n} \rightarrow \mathbb{R}$ and for the operator $\mathcal{A}_{l}$ defined by

$$
\mathcal{A}_{l} f(\mathbf{x})=\frac{1}{L(\mathbf{x})} \int_{\mathbb{R}_{+}^{n}} l\left(\frac{\mathbf{y}}{\mathbf{x}}\right) f(\mathbf{y}) d \mathbf{y}, \quad \mathbf{x} \in \mathbb{R}_{+}^{n} .
$$

Remark 7. Suppose that $l: \mathbb{R}_{+}^{n} \rightarrow \mathbb{R}$ is a non-negative measurable function such that $\int_{\mathbb{R}_{+}^{n}} l(\mathbf{y}) d \mathbf{y}=1$. Then, by setting $u(\mathbf{x}) \equiv 1$ and $r=1$, it follows that $v(\mathbf{y}) \equiv 1$, so inequality (28) reduces to

$$
\begin{aligned}
\int_{\mathbb{R}_{+}^{n}} \Psi & \left(\frac{1}{\mathbf{x}^{\mathbf{1}}} \int_{\mathbb{R}_{+}^{n}} l\left(\frac{\mathbf{y}}{\mathbf{x}}\right) f(\mathbf{y}) d \mathbf{y}\right) \frac{d \mathbf{x}}{\mathbf{x}^{\mathbf{1}}} \\
& +\int_{\mathbb{R}_{+}^{n}} \int_{\mathbb{R}_{+}^{n}} l\left(\frac{\mathbf{y}}{\mathbf{x}}\right) \Psi\left(\left|f(\mathbf{y})-\frac{1}{\mathbf{x}^{\mathbf{1}}} \int_{\mathbb{R}_{+}^{n}} l\left(\frac{\mathbf{y}}{\mathbf{x}}\right) f(\mathbf{y}) d \mathbf{y}\right|\right) \frac{d \mathbf{x}}{\mathbf{x}^{2 \mathbf{1}}} d \mathbf{y} \\
\leq & \int_{\mathbb{R}_{+}^{n}} \Psi(f(\mathbf{y})) \frac{d \mathbf{y}}{\mathbf{y}^{\mathbf{1}}}
\end{aligned}
$$

yielding an improved version of the multidimensional Godunova inequality from paper [8].

By virtue of Theorem 2, we can also derive multidimensional inequalities that correspond to those in Examples 1 and 2.

Example 3. Suppose $r$ and $\gamma$ are real parameters, such that $r \geq 1$ and $\gamma>1-\frac{1}{r}, \mathbf{0}<\mathbf{b} \leq \infty$ and $S_{1}^{n}=\left\{(\mathbf{x}, \mathbf{y}) \in \mathbb{R}^{n} \times \mathbb{R}^{n}: \mathbf{0}<\mathbf{y} \leq \mathbf{x}<\mathbf{b}\right\}$. Rewriting Theorem 2 with the weight function $u: \mathbb{R}_{+}^{n} \rightarrow \mathbb{R}, u(\mathbf{x})=\chi_{S_{1}^{n}}(\mathbf{x}, \mathbf{x})$, and with the kernel $l: \mathbb{R}_{+}^{n} \rightarrow \mathbb{R}, l(\mathbf{x})=\gamma^{n}(\mathbf{1}-\mathbf{x})^{(\gamma-1) \mathbf{1}} \chi_{(\mathbf{0}, \mathbf{1})}(\mathbf{x})$, it follows that $L(\mathbf{x})=\mathbf{x}^{\mathbf{1}}$, so we obtain the inequality

$$
\begin{aligned}
& \int_{(\mathbf{0}, \mathbf{b})} \Psi^{r}\left(\mathcal{R}_{\gamma, n} f(\mathbf{x})\right) \frac{d \mathbf{x}}{\mathbf{x}^{\mathbf{1}}} \\
& +r \gamma^{n} \int_{(\mathbf{0}, \mathbf{b})} \int_{(\mathbf{0}, \mathbf{x})}(\mathbf{x}-\mathbf{y})^{(\gamma-1) \mathbf{1}} \Psi^{r-1}\left(\mathcal{R}_{\gamma, n} f(\mathbf{x})\right) \Psi\left(\left|f(\mathbf{y})-\mathcal{R}_{\gamma, n} f(\mathbf{x})\right|\right) \frac{d \mathbf{x}}{\mathbf{x}^{(\gamma+1) \mathbf{1}}} d \mathbf{y} \\
& \leq\left(\int_{(\mathbf{0}, \mathbf{b})} w_{\gamma, n}(\mathbf{y}) \Psi(f(\mathbf{y})) \frac{d \mathbf{y}}{\mathbf{y}^{\mathbf{1}}}\right)^{r}
\end{aligned}
$$


where

$$
\mathcal{R}_{\gamma, n} f(\mathbf{x})=\frac{\gamma^{n}}{\mathbf{x}^{\gamma \mathbf{1}}} \int_{(\mathbf{0}, \mathbf{x})}(\mathbf{x}-\mathbf{y})^{(\gamma-1) \mathbf{1}} f(\mathbf{y}) d \mathbf{y}, \quad \mathbf{x} \in(\mathbf{0}, \mathbf{b})
$$

and

$$
w_{\gamma, n}(\mathbf{y})=\gamma^{n} \prod_{i=1}^{n} B^{\frac{p}{q}}\left(1-\frac{y_{i}}{b_{i}} ;(\gamma-1) \frac{q}{p}+1, \frac{q}{p}\right), \quad \mathbf{y} \in(\mathbf{0}, \mathbf{b}) .
$$

Clearly, $\mathcal{R}_{\gamma, n}$ represents an $n$-dimensional extension of the Riemann-Liouville operator $\mathcal{R}_{\gamma}$ defined by (19).

In particular, considering (29) with the superquadratic function $\Psi(x)=$ $x^{p}, p \geq 2$, the parameters $r=\frac{q}{p} \geq 1$ and $\mathbf{k} \in \mathbb{R}^{n}$, such that $\frac{\mathbf{k}-\mathbf{1}}{p \mathbf{1}}>\mathbf{0}$, and with $\mathbf{c}=\left(b_{1}^{\frac{k_{1}-1}{p}}, \ldots, b_{n}^{\frac{k_{n}-1}{p}}\right)$ and $f\left(y^{\frac{p}{k_{1}-1}}, \ldots, y_{n}^{\frac{p}{k_{n}-1}}\right) \mathbf{y}^{\frac{p 1}{\mathrm{k}-1}-\mathbf{1}}$, respectively instead of $\mathbf{b}$ and $f(\mathbf{y})$, after a sequence of suitable variable changes, we obtain the inequality

$$
\begin{aligned}
\int_{(\mathbf{0}, \mathbf{b})} & \mathbf{x}^{-\frac{q}{p}(\mathbf{k}-\mathbf{1})-\mathbf{1}}\left(\mathcal{R}_{n} f\right)^{q}(\mathbf{x}) d \mathbf{x} \\
+ & \frac{q}{p}\left(\frac{\gamma(\mathbf{k}-\mathbf{1})}{p \mathbf{1}}\right)^{\mathbf{1}} \int_{(\mathbf{0}, \mathbf{b})} \int_{(\mathbf{y}, \mathbf{b})} \prod_{i=1}^{n}\left[1-\left(\frac{y_{i}}{x_{i}}\right)^{\frac{k_{i}-1}{p}}\right]^{\gamma-1}\left(\mathcal{R}_{n} f\right)^{q-p}(\mathbf{x}) \\
\quad & \left|\left(\frac{p \mathbf{1}}{\gamma(\mathbf{k}-\mathbf{1})}\right)^{\mathbf{1}} f(\mathbf{y})\left(\frac{\mathbf{y}}{\mathbf{x}}\right)^{1-\frac{\mathbf{k}-1}{p \mathbf{1}}}-\frac{1}{\mathbf{x}^{\mathbf{1}}} \mathcal{R}_{n} f(\mathbf{x})\right|^{p} \\
& \times \mathbf{x}^{(p-q-1) \frac{\mathbf{k}-\mathbf{1}}{p \mathbf{1}}+p \mathbf{1}-\mathbf{k}} \mathbf{y}^{\frac{\mathbf{k}-\mathbf{1}}{p \mathbf{1}}-\mathbf{1}} d \mathbf{x} d \mathbf{y} \\
\leq \gamma^{n} & \left(\frac{p \mathbf{1}}{\gamma(\mathbf{k}-\mathbf{1})}\right)^{\left(q+1-\frac{q}{p}\right) \mathbf{1}}\left(\int_{(\mathbf{0}, \mathbf{b})} w_{\gamma, \mathbf{k}, n}(\mathbf{y}) f^{p}(\mathbf{y}) d \mathbf{y}\right)^{\frac{q}{p}},
\end{aligned}
$$

including the weight function

$$
w_{\gamma, \mathbf{k}, n}(\mathbf{y})=\mathbf{y}^{p \mathbf{1}-\mathbf{k}} \prod_{i=1}^{n} B^{\frac{p}{q}}\left(1-\left(\frac{y_{i}}{b_{i}}\right)^{\frac{k_{i}-1}{p}} ;(\gamma-1) \frac{q}{p}+1, \frac{q}{p}\right), \mathbf{y} \in(\mathbf{0}, \mathbf{b}),
$$

and the operator

$$
\mathcal{R}_{n} f(\mathbf{x})=\int_{(\mathbf{0}, \mathbf{x})}\left\{\prod_{i=1}^{n}\left[1-\left(\frac{y_{i}}{x_{i}}\right)^{\frac{k_{i}-1}{p}}\right]\right\}^{\gamma-1} f(\mathbf{y}) d \mathbf{y}, \mathbf{x} \in(\mathbf{0}, \mathbf{b}) .
$$

The example below may be regarded as a dual form of the previous example. 
Example 4. Consider the real parameters $r$ and $\gamma$, such that $r \geq 1$ and $\gamma>1-\frac{1}{r}$. Further, let $\mathbf{0} \leq \mathbf{b}<\infty$ and $S_{2}^{n}=\left\{(\mathbf{x}, \mathbf{y}) \in \mathbb{R}^{n} \times \mathbb{R}^{n}: \mathbf{b}<\mathbf{x} \leq \mathbf{y}<\infty\right\}$. This time, Theorem 2, for $u(\mathbf{x})=\chi_{S_{2}^{n}}(\mathbf{x}, \mathbf{x})$ and $l(\mathbf{x})=\gamma^{n} \mathbf{x}^{-(\gamma+1) 1}(\mathbf{x}-$ $\mathbf{1})^{(\gamma-1) \mathbf{1}} \chi_{(\mathbf{1}, \infty)}(\mathbf{x})$, yields the inequality

$$
\begin{aligned}
& \int_{(\mathbf{b}, \infty)} \Psi^{r}\left(\mathcal{W}_{\gamma, n} f(\mathbf{x})\right) \frac{d \mathbf{x}}{\mathbf{x}^{\mathbf{1}}} \\
& +r \gamma^{n} \int_{(\mathbf{b}, \infty)} \int_{(\mathbf{x}, \infty)}(\mathbf{y}-\mathbf{x})^{(\gamma-1) \mathbf{1}} \Psi^{r-1}\left(\mathcal{W}_{\gamma, n} f(\mathbf{x})\right) \\
& \quad \times \Psi\left(\left|f(\mathbf{y})-\mathcal{W}_{\gamma, n} f(\mathbf{x})\right|\right) d \mathbf{x} \frac{d \mathbf{y}}{\mathbf{y}^{(\gamma+1) \mathbf{1}}} \\
& \leq\left(\int_{(\mathbf{b}, \infty)} \widetilde{w}_{\gamma, n}(\mathbf{y}) \Psi(f(\mathbf{y})) \frac{d \mathbf{y}}{\mathbf{y}^{\mathbf{1}}}\right)^{r},
\end{aligned}
$$

where

$$
\mathcal{W}_{\gamma, n} f(\mathbf{x})=\gamma^{n} \mathbf{x}^{\mathbf{1}} \int_{(\mathbf{x}, \infty)}(\mathbf{y}-\mathbf{x})^{(\gamma-1) \mathbf{1}} f(\mathbf{y}) \frac{d \mathbf{y}}{\mathbf{y}^{(\gamma+1) \mathbf{1}}}, \quad \mathbf{x} \in(\mathbf{b}, \infty),
$$

is the $n$-dimensional Weyl operator and

$$
\tilde{w}_{\gamma, n}(\mathbf{y})=\gamma^{n} \prod_{i=1}^{n} B^{\frac{p}{q}}\left(1-\frac{b_{i}}{y_{i}} ;(\gamma-1) \frac{q}{p}+1, \frac{q}{p}\right), \quad \mathbf{y} \in(\mathbf{b}, \infty) .
$$

In addition, by considering inequality (31) with the superquadratic power function $\Psi(x)=x^{p}, p \geq 2$, the parameters $r=\frac{q}{p} \geq 1$ and $\mathbf{k} \in \mathbb{R}^{n}$ such that $\frac{p \mathbf{1}}{\mathbf{1 - \mathbf { k }}}>\mathbf{0}$, and with $\mathbf{d}=\left(b_{1}^{\frac{1-k_{1}}{p}}, \ldots, b_{n}^{\frac{1-k_{n}}{p}}\right)$ and $f\left(y_{1}^{\frac{p}{1-k_{1}}}, \ldots, y_{n}^{\frac{p}{1-k_{n}}}\right) \mathbf{y}^{\frac{p \mathbf{1}}{\mathbf{1}-\mathbf{k}}+\mathbf{1}}$, respectively instead of $\mathbf{b}$ and $f(\mathbf{y})$, as in Example 3 we obtain a new strengthened dual form of the multidimensional Hardy inequality

$$
\begin{aligned}
\int_{(\mathbf{b}, \infty)} \mathbf{x}^{\frac{q}{p}(\mathbf{1}-\mathbf{k})-\mathbf{1}}\left(\mathcal{W}_{n} f\right)^{q}(\mathbf{x}) d \mathbf{x} \\
+\frac{q}{p}\left(\frac{\gamma(\mathbf{1}-\mathbf{k})}{p \mathbf{1}}\right)^{\mathbf{1}} \int_{(\mathbf{b}, \infty)} \int_{(\mathbf{b}, \mathbf{y})} \prod_{i=1}^{n}\left[1-\left(\frac{x_{i}}{y_{i}}\right)^{\frac{1-k_{i}}{p}}\right]^{\gamma-1}\left(\mathcal{W}_{n} f\right)^{q-p}(\mathbf{x}) \\
\quad \times\left|\left(\frac{p \mathbf{1}}{\gamma(\mathbf{1}-\mathbf{k})}\right)^{\mathbf{1}} f(\mathbf{y})\left(\frac{\mathbf{y}}{\mathbf{x}}\right)^{\mathbf{1}+\frac{\mathbf{1 - k}}{p \mathbf{1}}}-\frac{1}{\mathbf{x}^{\mathbf{1}}} \mathcal{W}_{n} f(\mathbf{x})\right|^{p} \\
\quad \times \mathbf{x}^{(q-p+1) \frac{\mathbf{1 - k}}{p \mathbf{1}}+p \mathbf{1}-\mathbf{k} \mathbf{y}^{-\frac{1-\mathbf{k}}{p \mathbf{1}}-\mathbf{1}} d \mathbf{x} d \mathbf{y}}
\end{aligned}
$$




$$
\leq \gamma^{n}\left(\frac{p \mathbf{1}}{\gamma(\mathbf{1}-\mathbf{k})}\right)^{\left(q+1-\frac{q}{p}\right) \mathbf{1}}\left(\int_{(\mathbf{b}, \infty)} \widetilde{w}_{\gamma, \mathbf{k}, n}(\mathbf{y}) f^{p}(\mathbf{y}) d \mathbf{y}\right)^{\frac{q}{p}}
$$

where

$$
\tilde{w}_{\gamma, \mathbf{k}, n}(\mathbf{y})=\mathbf{y}^{p \mathbf{1}-\mathbf{k}} \prod_{i=1}^{n} B^{\frac{p}{q}}\left(1-\left(\frac{b_{i}}{y_{i}}\right)^{\frac{1-k_{i}}{p}} ;(\gamma-1) \frac{q}{p}+1, \frac{q}{p}\right), \mathbf{y} \in(\mathbf{b}, \infty),
$$

and

$$
\mathcal{W}_{n} f(\mathbf{x})=\int_{(\mathbf{x}, \infty)}\left\{\prod_{i=1}^{n}\left[1-\left(\frac{x_{i}}{y_{i}}\right)^{\frac{1-k_{i}}{p}}\right\rfloor\right\}^{\gamma-1} f(\mathbf{y}) d \mathbf{y}, \mathbf{x} \in(\mathbf{b}, \infty)
$$

Remark 8. Observe that Examples 3 and 4 respectively represent multidimensional extensions of Examples 1 and 2. Moreover, by setting $\gamma=1$ and $p=q$, inequalities (30) and (32) reduce to the corresponding relations from paper [20].

\section{A Class of Refined Hardy-Hilbert-Type Inequalities}

This section is dedicated to a refined class of the Hardy-Hilbert-type inequalities arising from our central result, that is, from Theorem 1. It is well-known that the classical Hardy-Hilbert inequality (6) is equivalent to the Hilbert inequality

$$
\int_{0}^{\infty} \int_{0}^{\infty} \frac{f(x) g(y)}{x+y} d x d y \leq \frac{\pi}{\sin \frac{\pi}{p}}\left(\int_{0}^{\infty} f^{p}(x) d x\right)^{\frac{1}{p}}\left(\int_{0}^{\infty} g^{p^{\prime}}(y) d y\right)^{\frac{1}{p^{\prime}}},
$$

where $p>1$ and $f \in L^{p}\left(\mathbb{R}_{+}\right), g \in L^{p^{\prime}}\left(\mathbb{R}_{+}\right)$are non-negative functions (for more details see [11]). In paper [14], the mentioned equivalence was established in a more general manner. More precisely, paper [14] provides a unified treatment of the Hilbert-type and the Hardy-Hilbert-type inequalities with conjugate exponents. We refer here only to the Hardy-Hilbert-type inequality involving a general non-negative homogeneous kernel $k$ of degree $-s, s>0$. Namely, if $p, p^{\prime}>1$ are conjugate exponents, then the inequality

$$
\int_{0}^{\infty} x^{(p-1)(s-1)+p\left(A_{1}-A_{2}\right)}\left(\int_{0}^{\infty} k(y, x) f(y) d y\right)^{p} d x
$$




$$
\leq k^{p-1}\left(2-s-p^{\prime} A_{1}\right) k\left(p A_{2}\right) \int_{0}^{\infty} x^{1-s+p\left(A_{1}-A_{2}\right)} f^{p}(x) d x
$$

holds, where $k(\alpha)=\int_{0}^{\infty} k(1, t) t^{-\alpha} d t$ and $p^{\prime} A_{1}, p A_{2} \in(1-s, 1)$.

Having in mind the form of the general integral operator $\mathcal{A}_{k}$ considered in this paper, it will be more convenient to rewrite (33) in a slightly altered form. Namely, replacing $k(y, x)$ with $k(x, y)$, relation (33) transforms to

$$
\begin{aligned}
& \int_{0}^{\infty} x^{(p-1)(s-1)+p\left(A_{1}-A_{2}\right)}\left(\mathcal{H}_{k} f\right)^{p}(x) d x \\
& \quad \leq k^{p-1}\left(p^{\prime} A_{1}\right) k\left(2-s-p A_{2}\right) \int_{0}^{\infty} x^{1-s+p\left(A_{1}-A_{2}\right)} f^{p}(x) d x,
\end{aligned}
$$

where $\mathcal{H}_{k}$ is the corresponding Hilbert-type operator, that is,

$$
\mathcal{H}_{k} f(x)=\int_{0}^{\infty} k(x, y) f(y) d y, \quad x>0 .
$$

Our first step in improving inequality (34) via superquadraticity consists in establishing a consequence of Theorem 1 related to a non-negative homogeneous kernel $k: \mathbb{R}_{+}^{2} \rightarrow \mathbb{R}$ of a negative real degree. For such homogeneous function $k$, we define

$$
k_{r}(\alpha)=\int_{0}^{\infty} k^{r}(1, t) t^{-\alpha} d t
$$

where $r \geq 1$ and $\alpha \in \mathbb{R}$ is such that $k_{r}(\alpha)<\infty$. If $r=1$, then $k_{r}(\alpha)$ will be denoted by $k(\alpha)$, as above.

Remark 9. Observe that, in definition (36), we consider simply all real arguments $\alpha$ for which $k_{r}(\alpha)$ converges, regardless their structure. On the other hand, inequalities (33) and (34) hold under a stronger condition, since it was assumed that the integral $k(\alpha)$ converges for all $\alpha \in(1-s, 1)$, where $-s$ is degree of homogeneity of the kernel $k$. Such condition was motivated by the fact that if $k: \mathbb{R}_{+}^{2} \rightarrow \mathbb{R}$ is strictly decreasing in each argument, then $(1-s, 1)$ covers all arguments $\alpha$ for which $k(\alpha)$ may converge (for more details see [6]).

Now, applying our Theorem 1 to the above setting, we obtain the main result regarding a non-negative homogeneous kernel of an arbitrary negative real degree and non-conjugate exponents.

Theorem 3. Let $p, q \in \mathbb{R}$ be such that $q \geq p \geq 2$ and let $k: \mathbb{R}_{+}^{2} \rightarrow \mathbb{R}$ be a non-negative homogeneous function of degree $-s, s>0$. If the parameters $a, b \in \mathbb{R}$ are such that $0<k(a)<\infty$ and $k_{\frac{q}{p}}\left(\frac{q}{p}(a-1)+b+2\right)<\infty$, then the 
inequality

$$
\begin{aligned}
& \int_{0}^{\infty} x^{b+q(a+s-1)}\left(\mathcal{H}_{k} f\right)^{q}(x) d x \\
& \quad+\frac{q}{p k(a)} \int_{0}^{\infty} \int_{0}^{\infty} k(x, y)\left(\mathcal{H}_{k} f\right)^{q-p}(x) \\
& \quad \times\left|k(a) f(y) y^{a}-x^{a+s-1} \mathcal{H}_{k} f(x)\right|^{p} x^{b+(a+s-1)(q-p+1)} y^{-a} d x d y \\
& \leq k^{\frac{q}{p^{\prime}}}(a) k_{\frac{q}{p}}\left(\frac{q}{p}(a-1)+b+2\right)\left(\int_{0}^{\infty} y^{\frac{p}{q}(b+1)+p a-1} f^{p}(y) d y\right)^{\frac{q}{p}}
\end{aligned}
$$

holds for all non-negative measurable functions $f: \mathbb{R}_{+} \rightarrow \mathbb{R}$ and the operator $\mathcal{H}_{k}$ defined by $(35)$.

Proof. To prove this theorem, we consider Theorem 1, that is, relation (11) with $k(x, y) y^{-a}$ and $f(y) y^{a}$, respectively instead of $k(x, y)$ and $f(y)$, as well as with $r=\frac{q}{p}, \Psi(x)=x^{p}$, and $u(x)=x^{b}$. Then we have $K(x)=k(a) x^{1-s-a}$,

$$
v(y)=k_{\frac{q}{p}}^{\frac{p}{q}}\left(\frac{q}{p}(a-1)+b+2\right) k^{-1}(a) y^{\frac{p}{q}(b+1)-1},
$$

and $\mathcal{A}_{k} f(x)=k^{-1}(a) x^{a+s-1} \mathcal{H}_{k} f(x)$, so (37) holds.

Next, we restate Theorem 3 for the case $p=q$. This result is interesting in its own right, since it will be utilized for obtaining a refinement of the HardyHilbert-type inequality (34).

Corollary 3. Let $p \in \mathbb{R}$ be such that $p \geq 2$ and let $k: \mathbb{R}_{+}^{2} \rightarrow \mathbb{R}$ be a non-negative homogeneous function of degree $-s, s>0$. If the parameters $a, b \in \mathbb{R}$ are such that $0<k(a)<\infty$ and $k(a+b+1)<\infty$, then the inequality

$$
\begin{aligned}
& \int_{0}^{\infty} x^{b+p(a+s-1)}\left(\mathcal{H}_{k} f\right)^{p}(x) d x \\
& \quad+\frac{1}{k(a)} \int_{0}^{\infty} \int_{0}^{\infty} k(x, y)\left|k(a) f(y) y^{a}-x^{a+s-1} \mathcal{H}_{k} f(x)\right|^{p} x^{b+a+s-1} y^{-a} d x d y \\
& \leq k^{p-1}(a) k(a+b+1) \int_{0}^{\infty} y^{b+p a} f^{p}(y) d y
\end{aligned}
$$

holds for all non-negative measurable functions $f: \mathbb{R}_{+} \rightarrow \mathbb{R}$ and the operator $\mathcal{H}_{k}$ defined by $(35)$.

The following consequence of Corollary 3 may be regarded as a new improvement of the Hardy-Hilbert-type inequality (34) for $p \geq 2$. 
Corollary 4. Let $p \in \mathbb{R}$ be such that $p \geq 2$ and let $k: \mathbb{R}_{+}^{2} \rightarrow \mathbb{R}$ be a non-negative homogeneous function of degree $-s, s>0$. If $A_{1}, A_{2} \in \mathbb{R}$ are such that $0<k\left(p^{\prime} A_{1}\right)<\infty$ and $k\left(2-s-p A_{2}\right)<\infty$, then the inequality

$$
\begin{aligned}
& \int_{0}^{\infty} x^{(p-1)(s-1)+p\left(A_{1}-A_{2}\right)}\left(\mathcal{H}_{k} f\right)^{p}(x) d x \\
& \quad+\frac{1}{k\left(p^{\prime} A_{1}\right)} \int_{0}^{\infty} \int_{0}^{\infty} k(x, y)\left|k\left(p^{\prime} A_{1}\right) f(y) y^{p^{\prime} A_{1}}-x^{p^{\prime} A_{1}+s-1} \mathcal{H}_{k} f(x)\right|^{p} \\
& \quad \times x^{-p A_{2}} y^{-p^{\prime} A_{1}} d x d y \\
& \leq k^{p-1}\left(p^{\prime} A_{1}\right) k\left(2-s-p A_{2}\right) \int_{0}^{\infty} x^{1-s+p\left(A_{1}-A_{2}\right)} f^{p}(x) d x
\end{aligned}
$$

holds for all non-negative measurable functions $f: \mathbb{R}_{+} \rightarrow \mathbb{R}$ and the operator $\mathcal{H}_{k}$ defined by $(35)$.

Proof. Follows easily from (38). Namely, setting $b+p a=1-s+p\left(A_{1}-A_{2}\right)$ implies that $b+p(a+s-1)=(p-1)(s-1)+p\left(A_{1}-A_{2}\right)$. Moreover, if $a=p^{\prime} A_{1}$, the above equation yields $b=1-s-p^{\prime} A_{1}-p A_{2}$, that is, $a+b+1=2-s-p A_{2}$, so we get (39).

Remark 10. In particular, if parameters $A_{1}$ and $A_{2}$ fulfill the relation

$$
p^{\prime} A_{1}+p A_{2}=2-s,
$$

then the inequality (39) reduces to

$$
\begin{aligned}
& \int_{0}^{\infty} x^{p(s-1)+p p^{\prime} A_{1}-1}\left(\mathcal{H}_{k} f\right)^{p}(x) d x \\
& +\frac{1}{k\left(p^{\prime} A_{1}\right)} \int_{0}^{\infty} \int_{0}^{\infty} k(x, y)\left|k\left(p^{\prime} A_{1}\right) f(y) y^{p^{\prime} A_{1}}-x^{1-p A_{2}} \mathcal{H}_{k} f(x)\right|^{p} \\
& \quad \times x^{-p A_{2}} y^{-p^{\prime} A_{1}} d x d y \\
& \leq k^{p}\left(p^{\prime} A_{1}\right) \int_{0}^{\infty} x^{p p^{\prime} A_{1}-1} f^{p}(x) d x .
\end{aligned}
$$

It should be noticed here that condition (40) was crucial in obtaining the best possible constant factors in the Hardy-Hilbert-type inequality (34) (see e.g. $[15])$.

In the sequel, we apply inequalities (39) and (41) to some particular kernels $k$. Such analysis will provide new improvements of some particular HardyHilbert-type inequalities known from the literature. 
Example 5. In Corollary 4, let the kernel $k$ be defined by $k(x, y)=$ $(x+y)^{-s}$, where $s>0$. Then $k(\alpha)=B(1-\alpha, s+\alpha-1)$, where $B$ is the usual Beta function, so $0<k(\alpha)<\infty$ for $1-s<\alpha<1$. Moreover, the Hilbert operator $\mathcal{H}_{k}$ reduces to the so-called Stieltjes transform of a non-negative function $f$, that is, to

$$
\mathcal{S} f(x)=\int_{0}^{\infty} \frac{f(y)}{(x+y)^{s}} d y, x \in \mathbb{R}_{+} .
$$

Further, the parameters $A_{1}=A_{2}=\frac{2-s}{p p^{\prime}}$, where $s>2-\min \left\{p, p^{\prime}\right\}$, fulfill relation (40). Hence, rewriting (41) in this setting, we have

$$
\begin{aligned}
& \int_{0}^{\infty} x^{(p-1)(s-1)}(\mathcal{S} f)^{p}(x) d x \\
& \quad+\frac{1}{\beta_{1}} \int_{0}^{\infty} \int_{0}^{\infty} \frac{\left|\beta_{1} f(y) y^{\frac{2-s}{p}}-x^{1+\frac{s-2}{p^{\prime}}} \mathcal{S} f(x)\right|^{p}}{(x+y)^{s}} x^{\frac{s-2}{p^{\prime}}} y^{\frac{s-2}{p}} d x d y \\
& \leq \beta_{1}^{p} \int_{0}^{\infty} x^{1-s} f^{p}(x) d x,
\end{aligned}
$$

where $\beta_{1}=B\left(\frac{p+s-2}{p}, \frac{p^{\prime}+s-2}{p^{\prime}}\right)$. Clearly, if $s=1$, then relation (42) reduces to the following improvement of the Hardy-Hilbert inequality (6), derived in paper [4]:

$$
\begin{gathered}
\int_{0}^{\infty}(\mathcal{S} f)^{p}(x) d x+\frac{\sin \frac{\pi}{p}}{\pi} \int_{0}^{\infty} \int_{0}^{\infty} \frac{\left|\frac{\pi}{\sin \frac{\pi}{p}} f(y) y^{\frac{1}{p}}-x^{\frac{1}{p}} \mathcal{S} f(x)\right|^{p}}{x+y} x^{-\frac{1}{p^{p}}} y^{-\frac{1}{p}} d x d y \\
\leq\left(\frac{\pi}{\sin \frac{\pi}{p}}\right)^{p} \int_{0}^{\infty} f^{p}(x) d x
\end{gathered}
$$

On the other hand, considering (41) with the same Stieltjes kernel $k$ and with the parameters $A_{1}=\frac{2-s}{2 p^{\prime}}, A_{2}=\frac{2-s}{2 p}$, we obtain the inequality

$$
\begin{aligned}
\int_{0}^{\infty} x^{\frac{s p}{2}-1}(\mathcal{S} f)^{p}(x) d x & \\
& +\frac{1}{\beta_{2}} \int_{0}^{\infty} \int_{0}^{\infty} \frac{\left|\beta_{2} f(y) y^{1-\frac{s}{2}}-x^{\frac{s}{2}} \mathcal{S} f(x)\right|^{p}}{(x+y)^{s}} x^{\frac{s}{2}-1} y^{\frac{s}{2}-1} d x d y \\
\leq & \beta_{2}^{p} \int_{0}^{\infty} x^{-\frac{s p}{2}+p-1} f^{p}(x) d x,
\end{aligned}
$$

where $\beta_{2}=B\left(\frac{s}{2}, \frac{s}{2}\right)$. It should be noticed here that relations (42) and (43) represent new refinements of the original Hardy-Hilbert-type inequalities derived in papers [23] and [24] (see also paper [13]). 
Example 6. For the function $k: \mathbb{R}_{+}^{2} \rightarrow \mathbb{R}$ given by $k(x, y)=\max \{x, y\}^{-s}$, where $s>0$, we denote the corresponding Hilbert-type operator by

$$
\mathcal{L} f(x)=\int_{0}^{\infty} \frac{f(y)}{\max \{x, y\}^{s}} d y, x \in \mathbb{R}_{+},
$$

and we have

$$
0<k(\alpha)=\frac{s}{(1-\alpha)(s+\alpha-1)}<\infty, \quad 1-s<\alpha<1 .
$$

Moreover, rewriting (41) with the above kernel and the parameters $A_{1}=A_{2}=$ $\frac{2-s}{p p^{\prime}}$, where $s>2-\min \left\{p, p^{\prime}\right\}$, we obtain the inequality

$$
\begin{aligned}
& \int_{0}^{\infty} x^{(p-1)(s-1)}(\mathcal{L} f)^{p}(x) d x \\
& \quad+\frac{1}{\lambda} \int_{0}^{\infty} \int_{0}^{\infty} \frac{\left|\lambda f(y) y^{\frac{2-s}{p}}-x^{1+\frac{s-2}{p^{\prime}}} \mathcal{L} f(x)\right|^{p}}{\max \{x, y\}^{s}} x^{\frac{s-2}{p^{\prime}}} y^{\frac{s-2}{p}} d x d y \\
& \quad \leq \lambda^{p} \int_{0}^{\infty} x^{1-s} f^{p}(x) d x
\end{aligned}
$$

where $\lambda=\frac{p p^{\prime} s}{(p+s-2)\left(p^{\prime}+s-2\right)}$. In particular, if $s=1$, then $\lambda=p p^{\prime}$, so (44) reduces to

$$
\begin{aligned}
\int_{0}^{\infty}(\mathcal{L} f)^{p}(x) d x+\frac{1}{p p^{\prime}} & \int_{0}^{\infty} \int_{0}^{\infty} \frac{\left|p p^{\prime} f(y) y^{\frac{1}{p}}-x^{\frac{1}{p}} \mathcal{L} f(x)\right|^{p}}{\max \{x, y\}} x^{-\frac{1}{p^{\prime}}} y^{-\frac{1}{p}} d x d y \\
& \leq\left(p p^{\prime}\right)^{p} \int_{0}^{\infty} f^{p}(x) d x
\end{aligned}
$$

yielding a refinement of the classical Hardy-Littlewood-Pólya inequality (for more details see [11]).

We finalize the previous discussion with another Hardy-Hilbert-type inequality, making use of the well-known reflection formula for the Digamma function $\psi$ (see e.g. [5]),

$$
\int_{0}^{\infty} \frac{\log t}{t-1} t^{-\alpha} d t=\psi^{\prime}(1-\alpha)+\psi^{\prime}(\alpha)=\frac{\pi^{2}}{\sin ^{2} \pi \alpha}, \alpha \in(0,1),
$$

and of the fact that

$$
Z(a, b)=\int_{0}^{\infty} t^{b} e^{-a t}\left(1-e^{-t}\right)^{-b} d t<\infty, a \in \mathbb{R}_{+}, b \geq 1 .
$$


More precisely, $Z(a, b)=\Gamma(b+1) \phi_{b}^{*}(1, b+1, a)$, where $\phi_{\mu}^{*}$ is the so-called unified Riemann-Zeta function,

$$
\phi_{\mu}^{*}(z, s, a)=\frac{1}{\Gamma(s)} \int_{0}^{\infty} t^{s-1} e^{-a t}\left(1-z e^{-t}\right)^{-\mu} d t,
$$

where $\mu \geq 1, \operatorname{Re} a>0$ and either $|z| \leq 1, z \neq 1$ and $\operatorname{Re} s>0$, or $z=1$ and $\operatorname{Re} s>\mu$ (for more information regarding the unified Riemann-Zeta function, see e.g. [9]).

Example 7. Observe that the function $k: \mathbb{R}_{+}^{2} \rightarrow \mathbb{R}$, defined by $k(x, y)=$ $\frac{\log y-\log x}{y-x}$, is homogeneous of degree $s=-1$ and that $0<k(\alpha)=\frac{\pi^{2}}{\sin ^{2} \pi \alpha}<\infty$, $\alpha \in(0,1)$. Denoting the corresponding Hilbert-type operator by $\mathcal{M}$, that is,

$$
\mathcal{M} f(x)=\int_{0}^{\infty} \frac{\log y-\log x}{y-x} f(y) d y, x \in \mathbb{R}_{+},
$$

relation (39) now becomes

$$
\begin{aligned}
\int_{0}^{\infty} x^{p\left(A_{1}-A_{2}\right)}(\mathcal{M} f)^{p}(x) d x+\frac{\sin ^{2} p^{\prime} A_{1} \pi}{\pi^{2}} \int_{0}^{\infty} \int_{0}^{\infty} \frac{\log y-\log x}{y-x} \\
\quad \times\left|\frac{\pi^{2}}{\sin ^{2} p^{\prime} A_{1} \pi} f(y) y^{p^{\prime} A_{1}}-x^{p^{\prime} A_{1}} \mathcal{M} f(x)\right|^{p} x^{-p A_{2}} y^{-p^{\prime} A_{1}} d x d y \\
\leq \frac{\pi^{2 p}}{\sin ^{2(p-1)} p^{\prime} A_{1} \pi \sin ^{2} p A_{2} \pi} \int_{0}^{\infty} x^{p\left(A_{1}-A_{2}\right)} f^{p}(x) d x,
\end{aligned}
$$

provided that $p^{\prime} A_{1}, p A_{2} \in(0,1)$.

Examples 5, 6, and 7 were adjusted for obtaining refinements of inequality (34) regarding particular choices of the kernel $k$ and the real parameters $A_{1}$ and $A_{2}$. However, the same particular settings can be considered with respect to a more general result with non-conjugate exponents $p$ and $q$, that is, with respect to Theorem 3. This is the content of the following important remark with which we conclude this paper.

Remark 11. Theorem 3, that is, the general Hardy-Hilbert-type inequality (37) regarding a homogeneous kernel and non-conjugate exponents, can also be applied to particular cases considered in Examples 5, 6, and 7. We only give here the form of the constant factor on the right-hand side of (37), that is, of the constant $k^{\frac{q}{p}}(a) k_{\frac{q}{p}}\left(\frac{q}{p}(a-1)+b+2\right)$ for all three cases.

More precisely, if $k(x, y)=(x+y)^{-s}, s>0$, then $k_{r}(\alpha)=B(1-\alpha, r s+\alpha-1)$, so the constant factor on the right-hand side of (37) reads

$$
B^{\frac{q}{p^{\prime}}}(1-a, s+a-1) B\left(\frac{q}{p}(1-a)-b-1, \frac{q}{p}(s+a-1)+b+1\right),
$$


provided that $1-s<a<1$ and $0<q(s+a-1)+p(b+1)<q s$.

Further, for the kernel $k(x, y)=\max \{x, y\}^{-s}, s>0$, we have

$$
k_{r}(\alpha)=\frac{r s}{(1-\alpha)(r s+\alpha-1)}, \quad 1-r s<\alpha<1,
$$

and the corresponding constant factor becomes

$$
\left[\frac{s}{(1-a)(s+a-1)}\right]^{\frac{q}{p^{\prime}}} \cdot \frac{p q s}{[q(1-a)-p(b+1)][q(s+a-1)+p(b+1)]},
$$

under the same conditions as in the previous case.

Finally, if $k(x, y)=\frac{\log y-\log x}{y-x}$, then the constant factor $k_{r}(\alpha)$ can be expressed in terms of the unified Riemann-Zeta function, that is, as

$$
k_{r}(\alpha)=Z(1-\alpha, r)+Z(\alpha+r-1, r),
$$

where $r \geq 1$ and $1-r<\alpha<1$. Moreover, the constant factor on the right-hand side of (37) reads

$$
\left(\frac{\pi}{\sin \pi a}\right)^{\frac{2 q}{p^{\prime}}}\left[Z\left(\frac{q}{p}(1-a)-b-1, \frac{q}{p}\right)+Z\left(\frac{q a}{p}+b+1, \frac{q}{p}\right)\right],
$$

provided that $0<a<1$ and $0<q a+p(b+1)<q$.

\section{References}

[1] S. Abramovich, S. Banić, M. Matić, Superquadratic functions in several variables, J. Math. Anal. Appl. 137(2) (2007), 1444-1460.

[2] S. Abramovich, G. Jameson, G. Sinnamon, Refining of Jensen's inequality, Bull. Math. Soc. Sci. Math. Roumanie (N.S.) 47(95) (2004), 3-14.

[3] S. Abramovich, G. Jameson, G. Sinnamon, Inequalities for averages of convex and superquadratic functions, J. Inequal. Pure Appl. Math. 7(2) (2004), Art. 70.

[4] S. Abramovich, K. Krulić, J. Pečarić, L.-E. Persson, Some new refined Hardy type inequalities with general kernels and measures, Aequationes Math. 79 (2010), 157-172.

[5] M. Abramowitz, I. A. Stegun, Handbook of mathematical functions with formulas, graphs, and mathematical tables, National Bureau of Standards, Applied Math. Series 55, 4th printing, Washington 1965. 
[6] A. Čižmešija, M. Krnić, J. Pečarić, General Hilbert's inequality with nonconjugate parameters, Math. Inequal. Appl. 11 (2008), 237-269.

[7] E. K. Godunova, Inequalities based on convex functions, Izv. Vysh. Uchebn. Zaved. Matematika, 47 (1965), 45-53; English transl. in Amer. Math. Soc., Transl., II Ser, 88 (1970), 57-66.

[8] E. K. Godunova, Generalization of a two-parameter Hilbert inequality, (Russian) Izv. Vyš̌. Učebn. Zaved. Matematika 1967 54(1) (1967), 3539.

[9] S. P. Goyal, A. Parashar, Some results for unified Riemann-zeta function, Kyungpook Math. J. 42(1) (2002), 87-94.

[10] G. H. Hardy, Notes on some points in the integral calculus, LX. An inequality between integrals, Messenger of Math. 54 (1925), 150-156.

[11] G. H. Hardy, J. E. Littlewood, G. Pólya, Inequalities, $2^{\text {nd }}$ edition, Cambridge University Press, Cambridge, 1967.

[12] S. Kaijser, L.-E. Persson, A. Öberg, On Carleman and Knopps Inequalities, J. Approx. Theory 117 (2002), 140-151.

[13] M. Krnić, J. Pečarić, Hilbert's inequalities and their reverses, Publ. Math. Debrecen, 67 (2005), 315-331.

[14] M. Krnić, J. Pečarić, General Hilbert's and Hardy's inequalities, Math. Inequal. Appl. 8 (2005), 29-52.

[15] M. Krnić, G. Mingzhe, J. Pečarić, G. Xuemei, On the best constant in Hilbert's inequality, Math. Inequal. Appl. 8 (2005), 317-329.

[16] K. Krulić, J. Pečarić, L.-E. Persson, Some new Hardy type inequalities with general kernels, Math. Inequal. Appl. 12 (2009), 473-485.

[17] A. Kufner, L. Maligranda and L.-E. Persson, The Hardy Inequality - About its History and Some Related Results, Vydavatelský Servis Publishing House, Pilsen, 2007.

[18] A. Kufner and L.-E. Persson, Weighted Inequalities of Hardy Type, World Scientific Publishing Co, Singapore/ New Jersey/ London/Hong Kong, 2003. 
[19] J. A. Oguntuase, L.-E. Persson, Refinement of Hardy's inequalities via superquadratic and subquadratic functions, J. Math. Anal. Appl. 339 (2008), 1305-1312.

[20] J. A. Oguntuase, L.-E. Persson, E. K. Essel, B. A. Popoola, Refined Multidimensional Hardy type inequalities via superquadracity, Banach J. Math. Anal. 2 (2008), 129-139.

[21] B. Opic and A. Kufner, Hardy-type inequalities, Pitman Research Notes in Mathematics Series, 219., Longman Scientific \& Technical, Harlow, 1990.

[22] L.-E. Persson, J. A. Oguntuase, Refinement of Hardy's inequality for "all" p, in: K.Mikio and L. Maligranda (eds.), Proc. Inter. Symp. Banach Func. Spaces 2006 (ISBFS 2006), Kitakyushu, Japan, Publishers, 2008, 129-144.

[23] B. Yang, On Hilbert's integral inequality, J. Math. Anal. Appl. 220 (1998), 778-785.

[24] B. Yang, On a general Hardy-Hilbert's integral inequality, Chin. Ann. of Math. 21A (2000), 401-408. 